\title{
Clinical significance of fasting and postprandial lipemia in obese and non-obese subjects
}

\begin{tabular}{rr}
\hline Muzha M. Baqi * & Abdulqader A. Alnaqishbandi ** \\
\hline & Abstract
\end{tabular}

Background and objective: Postprandial lipemia is a risk marker for the presence or progression of cardiovascular disease. Identifying factors affecting lipoprotein metabolism in postprandial state is of interest in order to find ways for prevention of atherosclerosis and cardiovascular diseases. This study aimed to assess obesity measurements and to find out the relationship between them and postprandial lipemia and to determine postprandial lipemia in obese subjects and to compare it with fasting lipemia taking in consideration the levels of Apolipoprotein B.

Methods: A cross-sectional study was designed to examine the association between obesity and postprandial lipemia. One hundred seventy one subjects of both genders with different body mass index participated in the study. Anthropometric measurements and blood pressure were recorded for them. Lipid profile was measured twice: after 12 hours fasting, two hours postprandially.

Results: There were significant differences between different groups of patients regarding with studied parameters. Men had a higher postprandial response than did women. There were significant differences between fasting and postprandial levels of lipid profile, except for the measurement of apolipoprotein B. Therefore, the level of plasma apolipoprotein B was not affected by meal intake. The postprandial lipemia was significantly related to the measurements of obesity.

Conclusion: Apolipoprotein B can replace low-density-lipoprotein-cholesterol in lipid profile assessments. By designing equations, by doing the postprandial triglyceride test, the level of fasting plasma triglyceride and level of plasma Apolipoprotein B can be estimated.

Keywords: Postprandial lipemia; Apolipoprotein B; Metabolic Syndrome.

\section{Introduction}

Cardiovascular disease (CVD) is regarded as one of the causes of morbidity and mortality worldwide nowadays. The incidence of this disease is likely to increase. Multiple risk factors contribute to CVD. Postprandial lipemia (PPL) represents the increment in lipid levels in plasma following ingestion of meal. ${ }^{1-5}$ This process is associated with an increase in very low-density lipoproteins (VLDL) that are transformed to low-density lipoprotein (LDL) and delivered to the arterial walls and a decrease of high-density lipoprotein (HDL) particle number and function, and production of small, dense LDL. This unfavorable lipid profile increases progression of atherosclerosis. ${ }^{6-8}$ Most of the people spend the majority of their time in the fed state, with a continual fluctuation in the degree of lipemia throughout the day. According to typical American eating pattern, most people consume three or more meals per day. Each of these meals is mostly consumed before the plasma triglyceride levels returned to baseline levels, this produces a lipemic condition resulting from the previous meal intake. ${ }^{9}$ Presence of high levels of lipids in postprandial state and impaired clearance

* Department of Laboratory, Kurdistan Medical Control Agency, Erbil, I raq

** Department of Clinical Analysis, College of Pharmacy, Hawler Medical University, Erbil, I raq. 
of postprandial lipoproteins have a closer relationship with atherosclerosis than levels in the fasting state. ${ }^{10-12}$ According to the findings from several previous studies, $40 \%$ of all patients with the premature coronary artery disease have normal fasting plasma lipids, while they suffer from impaired clearance of postprandial lipoproteins. ${ }^{13}$ The postprandial lipoprotein metabolism is affected by several factors including dietary pattern, food composition, other conditions associated with lifestyle, physiological factors (age, gender, genetic background and postmenopausal status) and cardiometabolic conditions. ${ }^{14}$ Studying the postprandial response can show multiple aspects of metabolic health that would not be noticeable from studying the fasting parameters. Although, fasting lipid and lipoprotein levels reflect steady state lipid metabolism even healthy subject is in a state of postprandial hypertriglyceridemia most of the time due to meal frequency. ${ }^{15}$ Also, it would be much simpler for patients if a random non-fasting sample could be used. ${ }^{16}$ The magnitude of PPL is increased by several risk factors including metabolic disorders. However, little is known about the relevance of postprandial lipemia in metabolic syndrome (MetS). ${ }^{17}$ In individuals likely to have excess abdominal fat, the National Health and Nutrition Examination Survey III committee recommended five main parameters in order to identify individuals with MetS. These five parameters consist of waist circumference, triglycerides (TGs), HDL-C, fasting glycemia, and blood pressure (BP). The committee prioritizes waist circumference over the body mass index (BMI) to estimate the amount of abdominal fat because abdominal obesity has been recognized as the most prevalent form of MetS in affluent, sedentary populations. Also, it recognized the importance of elevated TGs and reduced HDL-C concentrations as useful lipid markers of the presence of an atherogenic "Dysmetabolic" profile related to visceral obesity and ectopic fat deposition. Trying to identify individuals with aggregation of risk factors provides additional useful information to guide clinical management. ${ }^{18,19}$ This study aimed to determine PPL in obese subjects and to compare it with fasting lipemia taking into consideration the levels of Apolipoprotein $B$ (ApoB).

\section{Methods}

This cross-sectional study conducted in Rizgary Hospital (Outpatient Department) and private clinic in Erbil city, Kurdistan Region- Iraq, from September 2014 to January 2015. After taking permission from Hawler Medical University Ethics Committee for conducting this study and after signing on written consent, the study was done on 171 subjects with different BMI. All subjects were studied in the morning after 12 hours overnight fast. An ordinary meal was consumed, lipid profile measured in the fasting and 2 hours after the meal. The meal was containing a sandwich of fried meat, fried potato and pieces of uncooked tomato. In both samples which taken from them, uric acid, total cholesterol (TC), TG, HDL-c, LDL-C and $A p o B$ were measured. Both genders were included, aged between 35-65 years. All subjects had no symptoms of illness, and normal values for BP, blood creatinine, urea, and activities of aspartate (AST) or alanine (ALT) aminotransferase. None had any endocrine or gastrointestinal disease. Participants were interviewed and informed about the aim and nature of the study and then written consent was obtained from each subject. Subjects with renal disease, chronic liver disease, pregnancy, Diabetes Mellitus and hormonal disturbances were excluded from the study. Blood analysis was done for serum urea, creatinine, AST, ALT, thyroid stimulating hormone (TSH), free thyroxine (FT4), glycated hemoglobin $(\mathrm{HbA} 1 \mathrm{c})$, uric acid. Lipid profile included TC, TG, HDL-c, direct LDL-C and ApoB measurements. The instrument used for the measurement of biochemical tests, including $\mathrm{HbA} 1 \mathrm{c}, \mathrm{ALT}, \mathrm{AST}$, urea, uric acid, TG, glucose, creatinine, LDL-C, 
HDL-c, TC and ApoB, was the clinical chemistry analyzer cobas e311 Autoanalyzer machine (Roche Diagnostic $\mathrm{GmbH}$ D-68298, Mannheim, Germany). The instrument used for measurement of FT4 and TSH was cobas e 411 analyzer (Roche Diagnostic GmbH D-68298, Mannheim, Germany). According to their BMI, subjects were subdivided into obese subjects (30 or higher), overweight (25- 29.9) subjects, and within normal BMI (18.5- 24.9). Obese subjects were further classified into Class I (BMI $\geq 30)$, Class II $(B M I \geq 35)$ and Class III (BMI $\geq 40)$. Waist to hip ratio (WHR) was calculated and used as a measure of visceral obesity, which was defined as WHR $\geq 0.85$ for women and WHR $\geq 0.9$ for men. ${ }^{20-22}$ Waist to height ratio ( $\mathrm{WHtR})$ also measured as a measure of visceral obesity. It was calculated by dividing waist to height and the value of $\geq 0.5$ was regarded as at risk patients. ${ }^{20-22}$ Venous blood was sampled from the antecubital vein and collected into tubes with no anticoagulant and ethylene diamine tetraacetic acid (EDTA). Serum and plasma were separated from whole blood by centrifugation (5 min, 4100 rpm). Data were analyzed using the statistical package for the social sciences (version 19). Student's t-test was used to compare between means of two groups (independent samples t-test). Paired t-test was used to compare values of fasting and postprandial lipids. Multiple regression was done where the dependent variable was ApoB (postprandial), and the independent variables were fasting TC, TG, HDL-c, and LDL-C. The correlation was used for finding the correlation between different criteria under the study. A $P$ value of $\leq 0.05$ was considered statistically significant.

\section{Results}

The Table 1 shows the means of anthropometric measurements by gender. This study included 171 subjects (58 males and 113 females). There was a significant difference between mean BMI of females (31.7 \pm 7.4) and mean BMl of males (27.8 \pm 5.7). There was a significant difference between mean WHR of females $(0.849 \pm 0.074)$ and mean WHR of males $(0.896 \pm 0.064)$. There was a significant difference between mean WHtR of females $(0.618 \pm 0.105)$ and mean WHtR of males $(0.560 \pm 0.078)$.

Table 1: Means of anthropometric measurements by gender.

\begin{tabular}{lccc}
\hline Anthropometric measurements & Females $(n=113)$ & Males $(n=58)$ & $P$ value \\
\hline BMI $\left(\mathrm{kg} / \mathrm{m}^{2}\right)$ & $31.7 \pm 7.4$ & $27.8 \pm 5.7$ & 0.0006 \\
Waist circumference $(\mathrm{cm})$ & $98.5 \pm 16.4$ & $97.8 \pm 13.1$ & 0.781 \\
Hip circumference $(\mathrm{cm})$ & $115.7 \pm 14.0$ & $108.9 \pm 10.5$ & 0.0014 \\
WHR & $0.849 \pm 0.074$ & $0.896 \pm 0.064$ & 0.0001 \\
WHtR & & & \\
\hline
\end{tabular}

The results are expressed as mean \pm SD . 
Table 2 shows measurements of lipid profile and $A p o B$ levels at fasting and postprandial states. As it is obvious, significant differences can be seen between fasting and postprandial levels of TC, TG, HDL-C and LDL-C measurements in both genders. There was no significant difference between fasting and postprandial levels of $A p o B$ in both genders. Table 3 shows the determination of glucose, uric acid and $\mathrm{HbA} 1 \mathrm{c}$ at fasting and postprandial states. There was a significant difference between fasting and postprandial glucose level in both genders.

Table 2: Measurements of lipid profile and ApoB levels at fasting and postprandial states.

\begin{tabular}{|c|c|c|c|c|c|}
\hline & \multicolumn{2}{|c|}{ Female $(n=113)$} & \multicolumn{2}{|c|}{ Male $(n=58)$} & \multirow[b]{2}{*}{$P$ value } \\
\hline & Fasting state & Postprandial & Fasting state & Postprandial & \\
\hline $\mathrm{TC}(\mathrm{mg} / \mathrm{dl})$ & $169.7 \pm 36.1$ & $\begin{array}{c}166.7 \pm 36.0 \\
(\neq p=0.5)\end{array}$ & $176.9 \pm 39.5$ & $\begin{array}{c}174.2 \pm 38.7 \\
(\ddagger p=0.7)\end{array}$ & \\
\hline $\mathrm{TG}(\mathrm{mg} / \mathrm{dl})$ & $94.9 \pm 45.2$ & $\begin{array}{c}133.0 \pm 67.9 \\
(\neq p<0.0001)\end{array}$ & $136.7 \pm 81.9^{*}$ & $\begin{array}{l}183.2 \pm 106.4 \\
(\ddagger \mathrm{p}=0.0095)\end{array}$ & $\begin{array}{l}*<0.0001 \\
\dagger=0.0002\end{array}$ \\
\hline HDL-c (mg/dl) & $47.5 \pm 12.9$ & $\begin{array}{l}45.1 \pm 12.3 \\
(\neq p=0.15)\end{array}$ & $39.4 \pm 9.6$ & $\begin{array}{l}37.3 \pm 9.8 \\
(\neq p=0.24)\end{array}$ & $\begin{array}{l}{ }^{*}<0.0001 \\
\dagger<0.0001\end{array}$ \\
\hline LDL-c (mg/dl) & $99.1 \pm 28.3$ & $\begin{array}{l}95.2 \pm 27.8 \\
(\neq p=0.29)\end{array}$ & $108.2 \pm 31.3$ & $\begin{array}{c}104.0 \pm 30.9 \\
(\neq p=0.46)\end{array}$ & \\
\hline ApoB (mg/dl) & $91.0 \pm 24.0$ & $\begin{array}{c}90.7 \pm 24.0 \\
(\neq p=0.563)\end{array}$ & $99.6 \pm 23.5$ & $\begin{array}{l}99.9 \pm 26.1 \\
(p=0.759)\end{array}$ & $\begin{array}{l}* 0.025 \\
\dagger 0.027\end{array}$ \\
\hline
\end{tabular}

${ }^{*}$ Difference between male and female in fasting state. †Difference between male and female in the postprandial state. $\ddagger$ Difference between fasting state and postprandial state for each corresponding value of each gender.

Table 3: Determination of glucose, uric acid and HbA1c.

\begin{tabular}{|c|c|c|c|c|c|}
\hline & \multicolumn{2}{|c|}{ Female $(n=113)$} & \multicolumn{2}{|c|}{ Male $(n=58)$} & \multirow[b]{2}{*}{$P$ value } \\
\hline & Fasting state & Postprandial & Fasting state & Postprandial & \\
\hline Glucose (mg/dl) & $93.8 \pm 8.6$ & $\begin{array}{l}106.4 \pm 26.1 \\
(\ddagger p<0.001)\end{array}$ & $95.1 \pm 7.3$ & $\begin{array}{c}104.1 \pm 20.2 \\
( \pm p=0.0009)\end{array}$ & $\begin{array}{l}{ }^{*} 0.765 \\
+0.643\end{array}$ \\
\hline HbA1c (\%) & $5.646 \pm 0.404$ & & $5.438 \pm 0.373$ & & ${ }^{*} 0.466$ \\
\hline Uric acid (mg/dl) & $4.526 \pm 1.138$ & $\begin{array}{c}4.580 \pm 1.157 \\
(\ddagger p=0.743)\end{array}$ & $6.025 \pm 1.09$ & $\begin{array}{c}5.987 \pm 1.087 \\
(\ddagger \mathrm{p}=0.618)\end{array}$ & ${ }^{*}<0.001$ \\
\hline
\end{tabular}

*Difference between male and female in fasting state. †Difference between male and female in the postprandial state. $\ddagger$ Difference between fasting state and postprandial state for each corresponding value of each gender. 
Table 4 describes the measurements of lipid profile levels in respect to the measurement of obesity. There was a significant difference between fasting TC levels of normal BMl females (138.1 \pm 26.1) and males (171.2 \pm 30.6). There was a significant difference between postprandial cholesterol levels in normal BMl females $(134.7 \pm 23.8)$ and males $(174.5 \pm 45.7)$. Also, there was a significant difference between fasting levels of TG, HDL-C and ApoB in normal BMI, overweight and obese females and males. There was a significant difference between postprandial levels of TG, HDL-C and ApoB in normal $\mathrm{BMI}$, overweight and obese females and males. Table 5 describes the measurements of $\mathrm{BP}$ with respect to the BMI.

Table 4: Serum lipid profile levels in respect to the measurement of obesity.

\begin{tabular}{|c|c|c|c|c|c|c|c|}
\hline \multicolumn{2}{|c|}{ Lipid profile } & \multicolumn{3}{|c|}{ Fasting } & \multicolumn{3}{|c|}{ Postprandial } \\
\hline & & Female & Male & $P$ value & Female & Male & $P$ value \\
\hline \multirow{3}{*}{$\begin{array}{l}\text { TC } \\
(\mathrm{mg} / \mathrm{dl})\end{array}$} & Normal BMI & $138.1 \pm 26.1$ & $171.2 \pm 30.6$ & $<0.001$ & $134.7 \pm 23.8$ & $169.5 \pm 30.6$ & $<0.001$ \\
\hline & Overweight & $163.3 \pm 30.1$ & $174.5 \pm 45.7$ & & $160.4 \pm 32.9$ & $171.9 \pm 44.8$ & \\
\hline & Obese & $183.3 \pm 33.0$ & $187.1 \pm 43.2$ & & $180.4 \pm 33.3$ & $183.2 \pm 42.3$ & \\
\hline \multirow{3}{*}{$\begin{array}{l}\text { TGs } \\
(\mathrm{mg} / \mathrm{dl})\end{array}$} & Normal BMI & $65.5 \pm 26.7$ & $126.1 .2 \pm 95.1$ & 0.007 & $96.8 \pm 40.5$ & $177.3 \pm 134.4$ & $<0.001$ \\
\hline & Overweight & $89.6 \pm 44.9$ & $143.4 \pm 81.4$ & 0.017 & $134.6 \pm 71.8$ & $194.9 \pm 96.0$ & 0.03 \\
\hline & Obese & $107.3 \pm 45.8$ & $144.1 \pm 64.5$ & 0.039 & $144.7 \pm 70.2$ & $178.9 \pm 74.28$ & \\
\hline \multirow{3}{*}{$\begin{array}{l}\text { HDL-c } \\
\text { (mg/dl) }\end{array}$} & Normal BMI & $50.5 \pm 16.5$ & $40.7 \pm 12.5$ & 0.031 & $47.6 \pm 16.6$ & $39.1 \pm 12.9$ & \\
\hline & Overweight & $47.2 \pm 8.8$ & $37.4 \pm 7.3$ & $<0.001$ & $44.4 \pm 8.6$ & $34.9 \pm 7.9$ & $<0.001$ \\
\hline & Obese & $46.7 \pm 13.0$ & $39.9 \pm 6.9$ & 0.0054 & $44.5 \pm 12.0$ & $37.4 \pm 6.2$ & 0.001 \\
\hline \multirow{3}{*}{$\begin{array}{l}\text { ApoB } \\
(\mathrm{mg} / \mathrm{dl})\end{array}$} & Normal BMI & $69.3 \pm 15.7$ & $96.7 \pm 21.8$ & $<0.001$ & $69.1 \pm 16.1$ & $96.3 \pm 22.3$ & $<0.001$ \\
\hline & Overweight & $85.1 \pm 21.1$ & $99.7 \pm 24.5$ & 0.047 & $84.9 \pm 22.1$ & $100.6 \pm 30.5$ & \\
\hline & Obese & $100.9 \pm 21.8$ & $103.6 \pm 25.4$ & & $100.6 \pm 21.5$ & $104.0 \pm 26.7$ & \\
\hline
\end{tabular}

Table 5: Measurements of the blood pressure in respect to the body mass index.

\begin{tabular}{lcccccc}
\hline & \multicolumn{3}{c}{ SBP $(\mathrm{mmHg})$} & \multicolumn{3}{c}{ DBP $(\mathrm{mmHg})$} \\
& Females & Males & $\boldsymbol{P}$ value & Females & DBP & $\boldsymbol{P \text { value }}$ \\
\hline Normal weight & $109.8 \pm 12.5$ & $126.2 \pm 19.7$ & 0.002 & $74.9 \pm 6.8$ & $76.7 \pm 7.8$ & \\
Overweight & $119.2 \pm 18.5$ & $132.6 \pm 18.9$ & 0.024 & $78.0 \pm 9.0$ & $86.4 \pm 12.3$ & 0.018 \\
Obese & $131.5 \pm 19.8$ & $140.5 \pm 14.0$ & 0.039 & $82.4 \pm 9.0$ & $86.9 \pm 7.1$ & $\dagger 0.037$ \\
\hline
\end{tabular}


Table 6 shows the correlation between different parameters under the study. It shows a comparison between TGs and $A p o B$ and their correlation with other parameters under the study. Pearson 2-tailed correlation showed a significant direct positive correlation $(r=0.962)$ between ApoB and LDL-C. Also, it shows that the correlation between WHR and postprandial TG is higher than the correlation present between $\mathrm{BMI}$ and postprandial TG. Figure $1[\mathrm{~A}]$ shows a significant $(P=0.05)$ positive correlation (0.2) between serum TG and BMl in females.

Table 6: Correlations between different parameters under the study.

\begin{tabular}{lcccc}
\hline Parameters & \multicolumn{2}{c}{ TG (mg/dl) } & \multicolumn{2}{c}{ ApoB (mg/dl) } \\
& Pearson Correlation & Sig, (2-tailed) & Pearson Correlation & Sig, (2-tailed) \\
\hline TC (F) & 0.319 & $<0.001$ & 0.929 & $<0.001$ \\
TG (F)0 & 0.871 & $<0.001$ & 0.466 & $<0.001$ \\
HDL (F) & 0.415 & $<0.001$ & 0.135 & 0.79 \\
LDL (F) & 0.332 & $<0.001$ & 0.957 & $<0.001$ \\
ApoB (F) & 0.367 & $<0.001$ & 0.975 & $<0.001$ \\
TC (PP) & 0.343 & $<0.001$ & 0.931 & $<0.001$ \\
TG (PP) & 1 & & 0.351 & $<0.001$ \\
BMI & 0.03 & 0.696 & 0.339 & $<0.001$ \\
Waist circum. & 0.167 & 0.029 & 0.446 & $<0.001$ \\
WHR & 0.315 & $<0.001$ & 0.396 & $<0.001$ \\
SBP & 0.26 & 0.001 & 0.394 & $<0.001$ \\
DBP & 0.306 & $<0.001$ & 0.397 & $<0.001$ \\
Glucose (F) & 0.219 & 0.004 & 0.046 & 0.55 \\
\hline
\end{tabular}

Correlation is significant at the 0.01 level (2-tailed).

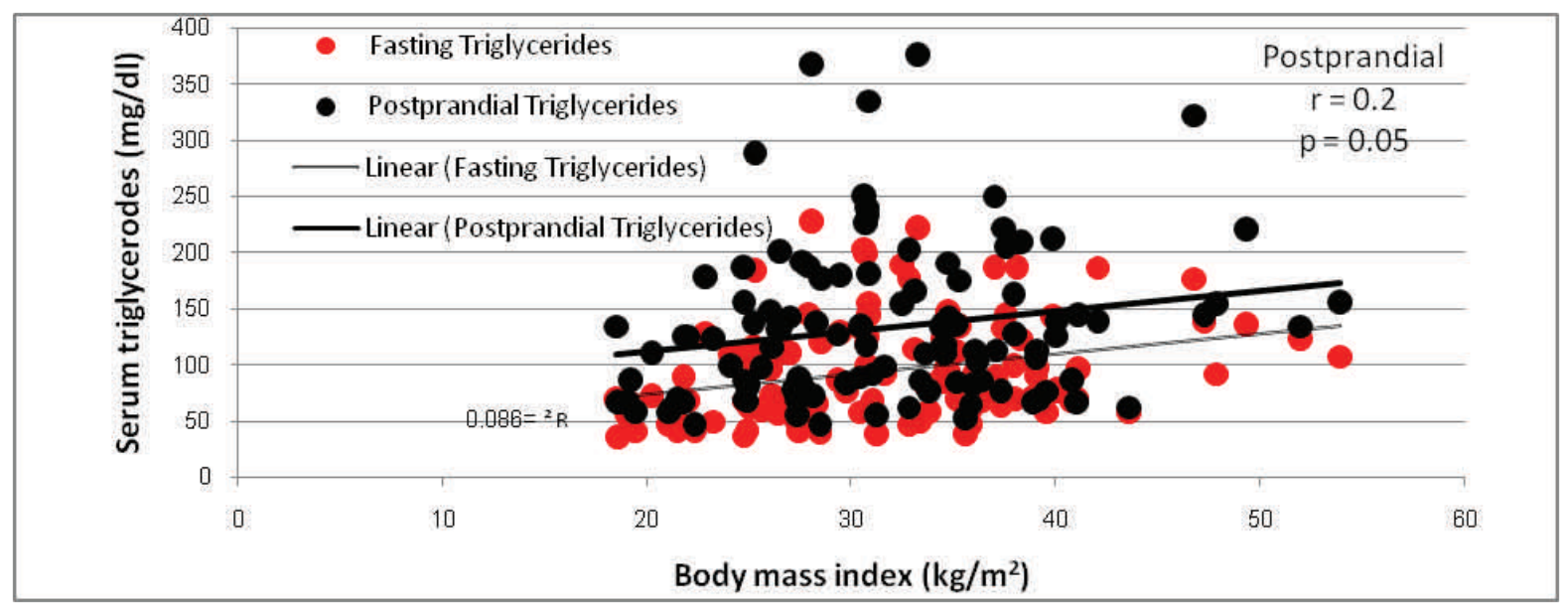

Figure 1[A]: Correlation between Serum triglycerides and body mass index in females. 
Figure 1[B] shows a non-significant correlation between serum TG and $\mathrm{BMI}$ in males. Figure 2 shows significant correlations between fasting and postprandial serum TGs in females $(r=0.85)$ and males $(r=0.869)$. The increment of postprandial TG depended on the fasting levels in both genders. Figure 3 shows a significant correlation between postprandial serum ApoB levels and TGs in both genders.

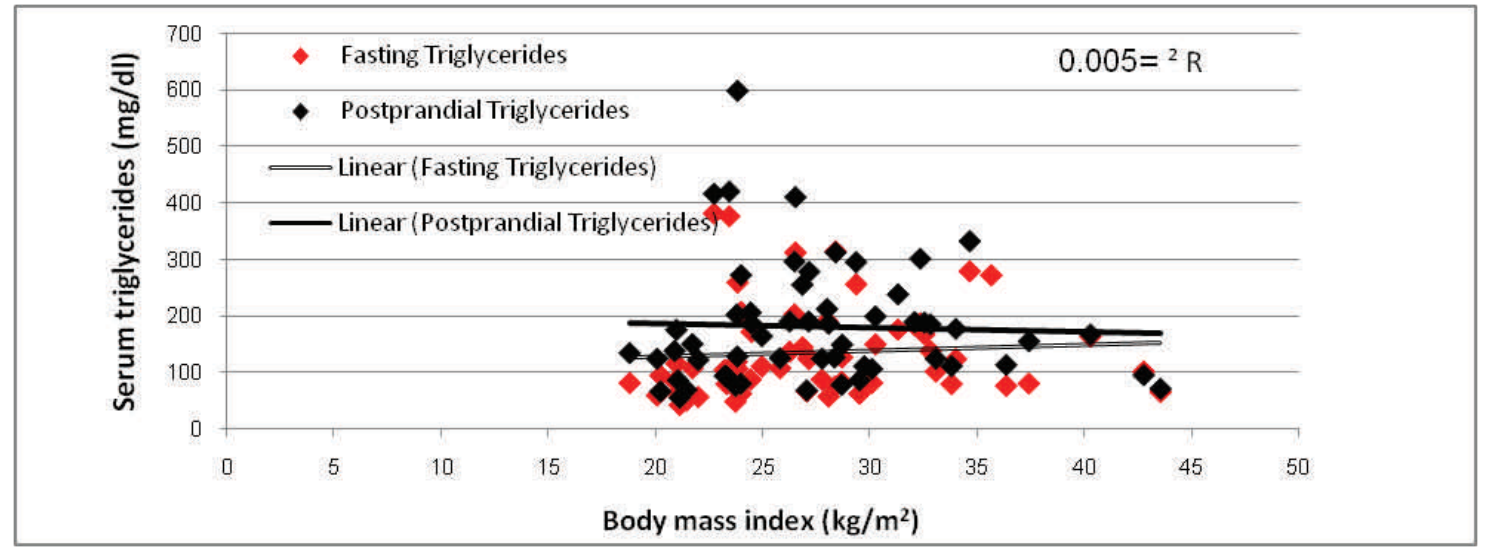

Figure1 [B]: Correlations between Serum triglycerides and body mass index in males.

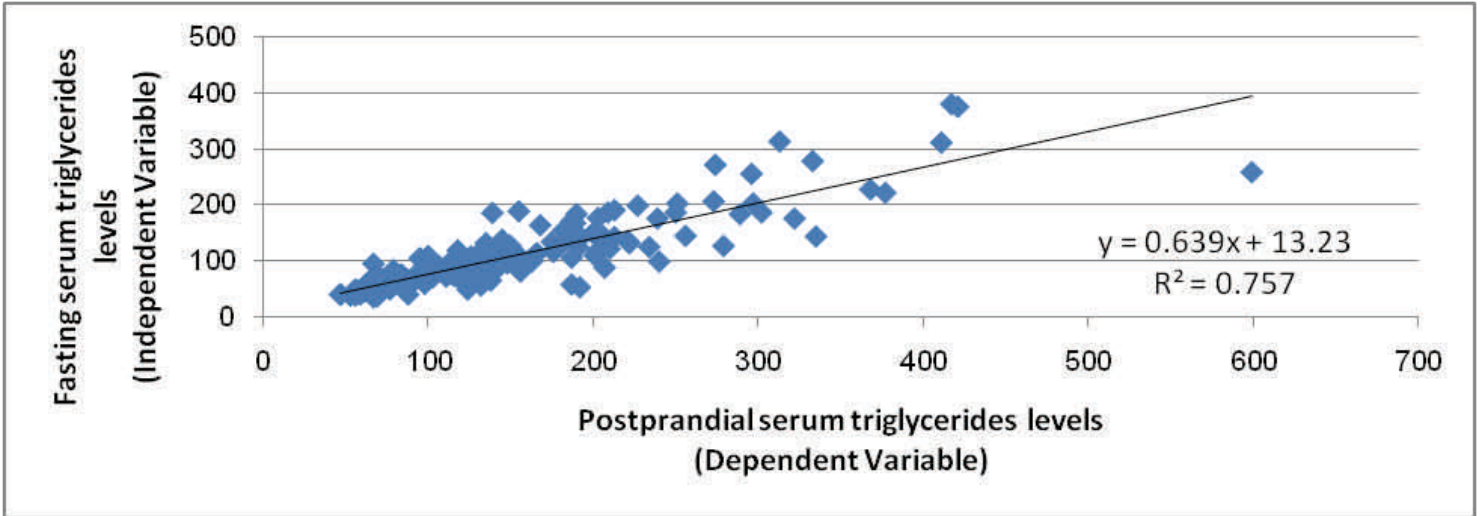

Figure 2: Correlation between fasting and postprandial serum triglycerides levels in both gender genders.

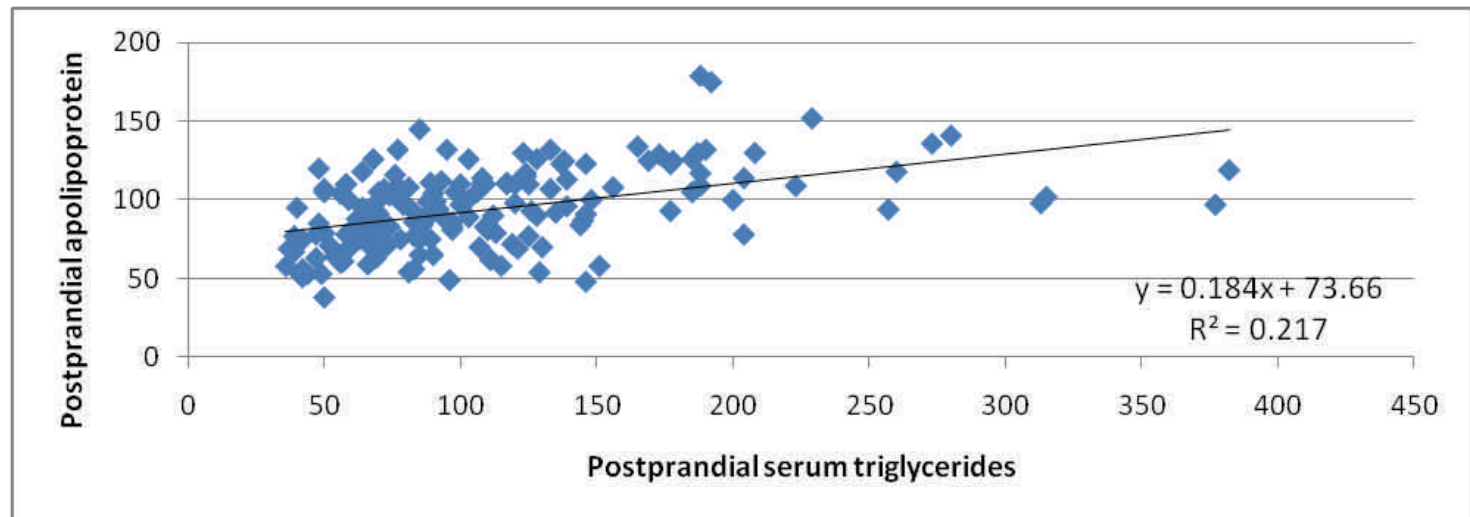

Figure 3: Correlation between postprandial serum apolipoprotein B levels and triglycerides in both genders. 
Figure 4 shows a significant correlation between fasting and postprandial ApoB in both genders.

\section{Discussion}

The finding was indicating that the largest body circumference for the women was around the hips and women are likely to have a lower body pattern of fat distribution as shown in Table 1, and men are known to present preferential accumulation of adipose tissue (AT) in the abdominal visceral depot and they are likely to have abdominal or upper-body obesity. ${ }^{20-22}$ Men had a higher ratio of WHR. Gluteofemoral fat deposition, an anatomic area of adiposity that has been shown to play a protective role in the development of cardiometabolic risk, Lower body obesity in women and accumulation of fat in these areas are results of hormonal difference between men and women, especially estrogen. This hormone has a cardioprotective effect. ${ }^{23-25}$ The results show a significant difference between fasting and postprandial state for the value of TC, TG, HDL-C and LDL-C, while the value of fasting and postprandial $A p o B$ is the same. The postprandial increase in TG is unequivocally (directly) due to the ingestion of meal. Decrease in the level of HDL-c in response to food intake is due to bidirectional lipid exchange between triglyceride-rich lipoprotein (TRL) and HDL particles. Decrease in the TC levels after food intake is a result of hemodilution state produced by food and fluid intake. ${ }^{16,26}$ Clinical assays of $A p o B$ measure both ApoB-100 and ApoB-48. Hence, total plasma ApoB (ApoB-100 + ApoB-48) represents the total atherogenic particle number. Therefore, measuring the number of atherogenic particles may be more biologically meaningful than the measuring cholesterol content in particles. ${ }^{27-30}$ The results show that men are characterized by an overall less favorable plasma lipid profile. Estrogens have been suggested to have a favorable impact on postprandial triglyceridemia. Variation in lipoprotein lipase activity between men and women may also be implicated in the gender difference in PPL. Once again, the greater accumulation of subcutaneous fat in women than in men could play a role in the gender difference noted in the clearance of TRL after a dietary fat challenge. Also estrogens are linked to higher HDL levels. It has been suggested that the higher fasting TG in men may in some way drive their higher postprandial TG response. ${ }^{17}$ The results show a significant difference between fasting and nonfasting blood glucose levels as shown in the Table 3, which is directly related to ingestion of food. ${ }^{26}$ Also, the serum uric acid levels were significantly higher in men compared to women. Uric acid measurement is

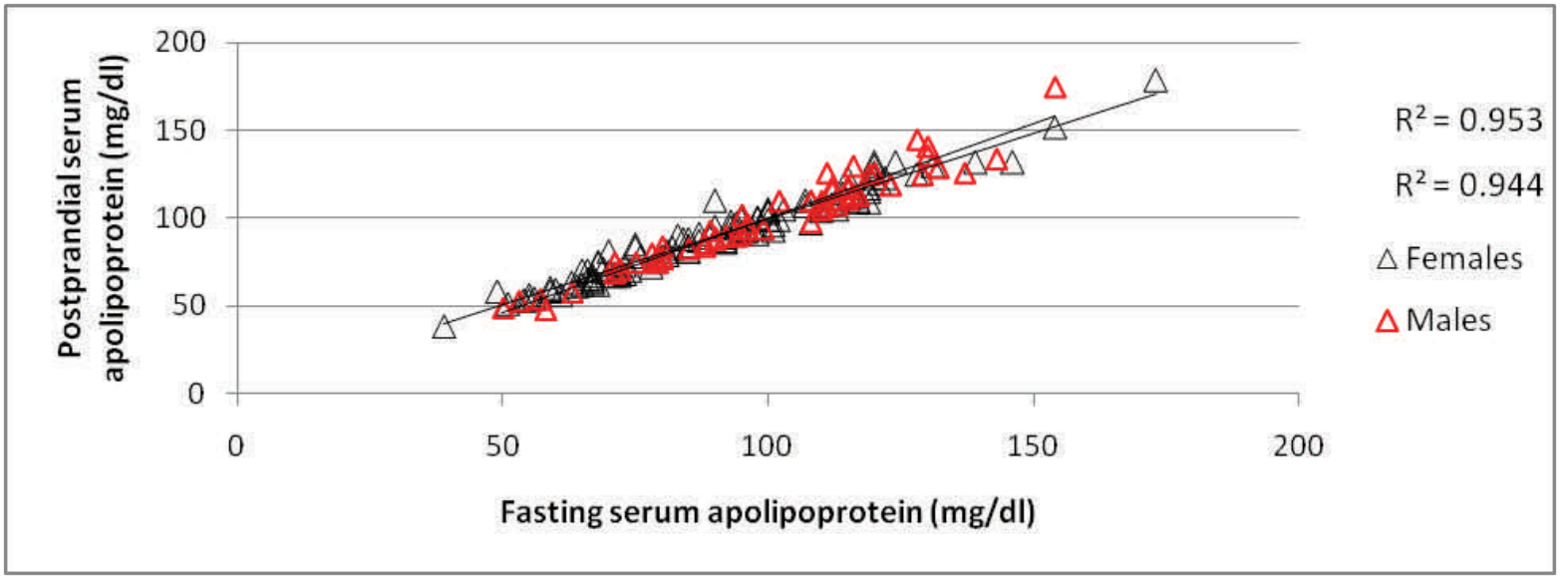

Figure 4: Correlations between fasting and postprandial apolipoprotein $B$ in both genders. (Female: $r=0.976, P<0.001$, Male: $r=0.0972, P<0.001$ ) 
of great importance because high levels mean good defense mechanism (because uric acid is a strong scavenger of nitrosative radical particularly peroxynitrite). ${ }^{22,31}$ The results in Table 4 show that with increasing BMI, level of fasting and postprandial TC, TG in the serum will be increased. More AT accumulation plays an important role in the PPL and this will lead to produce a higher postprandial TG response in obese subjects. ${ }^{13}$ Also the results show decrease in the HDL-c levels as BMI increased. HDL metabolism is also strongly affected by obesity because of the increased number of remnants of chylomicrons and VLDL together with impaired lipolysis. This will ultimately lead to lower levels of HDL-C and a reduction in circulating $\mathrm{HDL}$ particles with impairment of reversed cholesterol transport. $^{33}$ The results also show that obesity increases risk of hypertension, and it show that respondents with BMI $30 \mathrm{~kg} / \mathrm{m}^{2}$ or greater were likely to have hypertension compared with non-obese subjects and the results in Table 5 show that men have significant higher blood pressure in comparison to women. ${ }^{20}$ The results show significant positive direct correlation between serum TG and BMI in women as shown in the Figure $1[A]$. The correlation lines run in parallel; the line of postprandial shifted upward, which means the postprandial level increase so much compared with fasting for the same BMI. There is a non-significant correlation between serum TG and BMI in men as shown in the Figure $1[\mathrm{~B}]$. The correlation lines do not run in parallel; therefore, no correlation between $\mathrm{BMI}$ and TG can be defined in men. This indicates that there is no link between lipemia and BMI in men. Therefore, it can be estimated that there may be another factor rather than BMI influence the postprandial TG response in men. ${ }^{15}$ The results in Figure 2 show significant correlation between fasting and postprandial serum TGs in both genders. From this figure we can determine the fasting serum TG levels at any time of estimation the serum TGs (postprandial) whatever male or female by using this equation:

Fasting TG level $=(0.639 \times$ postprandial TG) + 13.236

It means that there is no need to ask the patient to be fasting for estimating TG level. There is a strong positive correlation $(r=0.871)$ between fasting TG and the postprandial plasma TG response. There are several previous studies which indicate that fasting TGs is the major contributor to the postprandial TG level. ${ }^{6,8,9}$ The results show a significant correlation between serum levels of $A p o B$ and TG postprandially. Therefore, it can be estimated that the serum level of $A p o B$ is dependent on the serum level of TG postprandially, by using a simple equation the changes in the TG can be determined which is corresponding to the changes in ApoB level:

Postprandial $\mathrm{ApoB}=(0.184 \times$ postprandial serum TGs) + 73.661

The results show a significant correlation between fasting and postprandial $A p o B$ in both genders as shown in Figure 4. There is no need to be fasting for estimating serum ApoB level and it can be estimated at any time of measurement and it gives the same result ${ }^{29,}{ }^{30}$. Multiple regression analysis showed that there was a strong correlation between $A p o B$ measurement and LDL-c measurement. Also, Pearson 2-tailed correlation showed significant direct positive correlation $(r=0.962)$ between ApoB and LDL-c. Recent data demonstrate that $A p o B$ is a better measure of circulating LDL particle number LDL-p concentration and is a more reliable indicator of risk than LDL-c. ${ }^{28}$

\section{Conclusion}

Postprandial hypertriglyceridemia which is the most clinically important part of postprandial lipemia is well related to the severity of obesity that assessed by BMI. The serum level of postprandial $A p o B$ does not significantly differ from the corresponding fasting levels. Therefore, 
it is possible to assess the levels of atherogenic lipid at any time without need fasting state. Moreover, determination of serum $A p o B$ gives us a clue about the status of TGs or LDL-C. Apolipoprotein B is a more reliable indicator of CVD than LDL-C and it is not affected by fasting. The response of PPL in obese individuals is a gender based effect, i.e., men are more vulnerable to cardiovascular events. PPL response is extended to include a significant increase serum levels of uric acid, which again this effect is gender-based. This study elaborates equations by which it can be possible to predict fasting levels of TGs from the postprandial levels. The correlation between WHR and postprandial TG is higher than the correlation present between $\mathrm{BMI}$ and postprandial TG.

\section{Competing interests}

The authors declare that they have no competing interests.

\section{References}

1. Ballantyne C, Arroll B, Shepherd J. Lipids and CVD management: towards a global consensus. Eur Heart J 2005; 26 (21):2224-31.

2. Yuan G, Al-Shali KZ, Hegele RA. Hypertriglyceridemia: its etiology, effects and treatment. CMAJ 2007; 176 (8):1113-20.

3. Freiberg JJ, Tybjaerg-Hansen A, Jensen JS, Nordestgaard BG. Nonfasting Triglycerides and Risk of Ischemic Stroke in the General Population. JAMA 2008; 300 (18):2142-52.

4. Ntyintyane LM, Panz VR, Raal FJ, Gill GV. Postprandial lipaemia, metabolic syndrome and LDL particle size in urbanised South African blacks with and without coronary artery disease. QJM 2008; 101 (2):111-9.

5. Stenger C, Hanse M, Pratte D, Mbala ML, Akbar S, Koziel V, et al. Up-regulation of hepatic lipolysis stimulated lipoprotein receptor by leptin: a potential lever for controlling lipid clearance during the postprandial phase. FASEB J 2010; 24 (11):4218-28.

6. Sandeep B, Julie EB, Rifai N, Samia M, Frank MS, Paul MR. Fasting Compared With Nonfasting Triglycerides and Risk of Cardiovascular Events in Women. JAMA 2007; 298(3):309-16.

7. Matsumoto S, Gotoh N, Hishinuma S, Abe Y, Shimizu Y, Katano Y, et al. The role of hypertriglyceridemia in the development of atherosclerosis and endothelial dysfunction. Nutrients $2014 ; 6(3): 1236-50$.
8. Paloma AV, Daniel CR, Roopa M, Liliana $\mathrm{MH}$, Ivette CB, Oscar PM, et al. Factors associated with postprandial lipemia and apolipoprotein A-V levels in individuals with familial combined hyperlipidemia. BMC Endocrine Disorders 2014; 14(1):43.

9. Ridker PM. Fasting versus nonfasting triglycerides and the prediction of cardiovascular risk: Do we need to revisit the oral triglyceride tolerance test? Clin Chem 2008; 54(1):11-3.

10. Zilversmit DB. Atherogenesis: a postprandial phenomenon. Circulation 1979; 60(3):473-85.

11. Zheng C, Ikewaki K, Walsh BW, Sacks FM. Metabolism of apoB lipoproteins of intestinal and hepatic origin during constant feeding of small amounts of fat. J Lipid Res 2006; 47(8):1771-9.

12. Parthasarathy S. Lipolysis Needed for Chylomicron Uptake? Arterioscler Thromb VascBiol 2010; 30:5-6.

13. Cox-York KA, Sharp TA, Stotz SA, Bessesen $\mathrm{DH}$, Pagliassotti MJ, Horton TJ. The effects of sex, metabolic syndrome and exercise on postprandial lipemia. Metabolism 2013; 62 (2): 244-54.

14. Juan FAD, Javier $\mathrm{DL}$, Pablo $\mathrm{PM}$, Antonio GR, Carmen M, Gracia MQN, et al. Hypertriglyceridemia Influences the Degree of Postprandial Lipemic Response in Patients with Metabolic Syndrome and Coronary Artery Disease: From the Cordioprev Study. PLoS ONE 2014; 9(5):e96297.

15. Nadia A, Boumediene KM. Effect of Overweight and Obesity on Postprandial Lipemia among the Subjects with Type 2 Diabetes. Diabetes Metab J 2012; 3(2):5.

16. Langsted A, Freiberg JJ, Nordestgaard BG. Fasting and nonfasting lipid levels: influence of normal food intake on lipids, lipoproteins, apolipoproteins, and cardiovascular risk prediction: Circulation 2008; 118(20):2047-56.

17. Garcia-Rios A, Delgado-Lista J, Perez-Martinez $\mathrm{P}$, Delgado-Casado N, Perez-Jimenez F, Lopez-Miranda J. Relevance of Postprandial Lipemia in Metabolic Syndrome. CurrVascPharmacol 2013; 11(6):920-7.

18. Grundy SM, Cleeman JI, Daniels SR, Donato KA, Eckel RH, Franklin BA, et al. Diagnosis and management of the metabolic syndrome: an American Heart Association/ National Heart, Lung, and Blood Institute scientific statement. Curr Opin Cardiol 2006; 21(1):1-6.

19. Després PJ, Poirier P, Bergeron J, Tremblay A, Lemieux I, Alméras N. From individual risk factors and the metabolic syndrome to global cardiometabolic risk. Europ Heart J Suppl 2008; 10(Supplement B):B24-33.

20. Janghorbani $M$, Amini $M$, Willett $W C$, Mehdi Gouya M, Delavari A, Alikhani S, et al. First nationwide survey of prevalence of overweight, underweight, and abdominal obesity 
in Iranian adults: Obesity (Silver Spring) 2007; 15 (11):2797-808.

21. Tsai AG, Williamson DF, Glick HA. Direct medical cost of overweight and obesity in the USA: a quantitative systematic review. Obes Rev 2011; 12(1):50-61.

22. Wong AT, Chan DC, Pang J, Watts GF, Barrett $\mathrm{PH}$. Plasma apolipoprotein B-48 transport in obese men: a new tracer kinetic study in the postprandial state. J Clin Endocrinol Metab 2014; 99(1):E122-6.

23. Freedman DS, Kahn HS, Mei Z, Grummer-Strawn LM, Dietz WH, Srinivasan SR, et al. Relation of body mass index and waist-toheight ratio to cardiovascular disease risk factors in children and adolescents: the Bogalusa Heart Study. Am J Clin Nutr 2007; 86(1):33-40.

24. Kumar P. A Hypothetical Index for Adiposity "Body Mass Abdominal Index"- That will predict Cardiovascular disease risk factors in Children. Rom J Pediatr 2010; 59(1):33-5.

25. Ulijaszek SJ, Henneberg M, Henry CJ. One reason why waist-to-height ratio is usually better related to chronic disease risk and outcome than body mass index [Abstract]. Int $\mathrm{J}$ Food SciNutr 2013; 64(3):269-73.

26. Schwander F, Kopf-Bolanz KA, Buri C, Portmann $R$, Egger $L$, Chollet $M$, et al. A Dose-Response Strategy Reveals Differences between NormalWeight and Obese Men in Their Metabolic and Inflammatory Responses to a High-Fat Meal. J Nutr 2014; 144(10):1517-23.

27. Mora S, Rifai N, Buring JE, Ridker PM. Fasting compared with nonfasting lipids and apolipoproteins for predicting incident cardiovascular events [Abstract]. Circulation 2008; 118(10):993-1001.

28. Contois JH, McConnell JP, Sethi AA, Csako G, Devaraj S, Hoefner DM, et al. Apolipoprotein B and cardiovascular disease risk: position statement from the AACC Lipoproteins and Vascular Diseases Division Working Group on Best Practices. Clin Chem 2009; 55(3):407-19.

29. Jacobson TA, Harper CR. Using Apolipoprotein B to Manage Dyslipidemia-Reply-I. Mayo Clin Proc 2010; 85 (8):769-71.

30. Chang JG, Paulson CP, Smith RF. Apolipoproteins for Cardiovascular Risk Assessment. Am Fam Physician 2014; 89 (8):662A-B.

31. Sautin YY, Johnson RJ. Uric acid: the oxidantantioxidant paradox. Nucleosides Nucleotides Nucleic Acids 2008; 27(6):608-19.

32. Samimi A, Ramesh Sh, Turin TC, MacRae JM, Sarna MA, Reimer RA, et al. Serum uric acid level, blood pressure, and vascular angiotensin II responsiveness in healthy men and women. Physiological Reports 2014: 2(12):e12235.

33. Boudewijn K, Jan WFE, Manuel CC. Dyslipidemia in Obesity: Mechanisms and Potential Targets. Nutrients 2013; 5(4):1218-40. 